\title{
L'espace et l'homme dans les romans de Michel Butor Une étude préparée par
}

\section{Amr Ali Gomaa}

Dès son premier roman, Butor se met à évoquer les procès et les souffrances de l'homme dans ce monde mobile et instable. Ce monde qui connaît des transmutations impétueuses et considérables conviennent aux mutations politiques et sociales qui frappent la société française après la seconde guerre mondiale et la chute militaire imprévue de la France, ces transformations qui influencent et menacent le concept de l'existence et de l'identité de l'homme.

Le rapport entre l'homme et son espace est une connexité notablement cohérente et infrangible, puisque l'espace a des impacts considérables sur les conduites et sur les relations entre les personnages. Ainsi, nous désirons ici de connaître quelle est l'influence de l'espace sur les personnages dans les romans de Michel Butor ?, et comment Michel Butor parvient-il au moyen de l'espace à représenter à son lecteur les souffrances de ses personnages?

En fait, le rapport entre l'espace et les personnages est une relation très importante pour le succès des œuvres butoriennes, puisqu'il a choisi soigneusement l'espace qui convient parfaitement aux conditions de ses personnages. Il implante ses personnages dans un espace urbain moderne. Ce milieu possède tous les moyens de communication moderne. En dépit de tous ces moyens, il nous présente ces personnages dans un isolement complet, ils vivent dans des îles isolées et dans des cases enfermées. Il est à noter que: "Chez Butor, les personnages seront éternellement enfermés dans ce cadre où toutes les intrigues seront nouées à l'intérieur. En fait, cette clôture prend une dimension symbolique puisqu'elle signifie celle des consciences cloitrées, bornées, aliénées des protagonistes résidant dans cet espace. Elle (la clôture) a aussi une fonction formelle, plutôt que de laisser l'inspiration se disposer à l'indéfini, Butor se sert de cet espace bien défini, bien déterminé et hermétiquement clos afin de bien cadrer son imagination romanesque. ${ }^{" 1}$

Débutons, par Passage de Milan, où il tend à incarner toutes les questions littéraires, sociales et politiques qui préoccupent les gens lors de cette phase : "Lorsqu'on travaille à son premier livre, (écrit Butor), on n'est jamais sûr qu'il sera publié, et surtout on n'est jamais sûr qu'il y en aura un autre. Par conséquent le jeune écrivain a une tendance à essayer de mettre le plus de choses possible dans ce premier livre, pour qu'il ait fait plus ou moins ce qu'il avait à faire, les premiers livres sont donc souvent touffus, et c'est le cas pour celui-ci, ce qui rend sa lecture assez difficile" ${ }^{\prime 2}$, c'est pourquoi, cette première tentative littéraire contient tous : "Les germes" ${ }^{" 3}$, principaux de tous ses romans postérieurs, ainsi que l'isolement, l'exil, la corruption morale et les fausses relations entre les habitants...etc.

Vu que dès la première page, Passage de Milan connaît l'enferment, l'exil et l'isolement de tous les habitants de cette maison. Dans le premier étage, chez les deux frères Jean et Alexis, l'enfermement et l'isolement règnent leurs relations. Cet exemple nous raconte la routine et les fausses relations entre les personnages de cet 
étage: "Il l'embrassa. Il claqua la porte derrière lui. Il tenait son chapeau à la main. Elle (Mme Virginie Ralon) le vit accrocher sa pèlerine au porte-manteau, pénétrer dans sa chambre sans dire un mot, scène qui se reproduisait tous les soirs, et déposait en elle chaque soir un peu plus de tristesse, car elle aurait bien voulu qu'Alexis au moins mit au courant de ses ennuis,..." 4

Restons au premier étage, mais cette fois, avec Mme Virginie Ralon qui parle à charlotte sur son fils Jean, qui vit dans une solitude totale : Car vous savez bien que pour Jean c'était naturel, si loin de moi déjà dans ses livres, perdu parmi toutes ces choses bizarres qui l'occupent et le rendent pensif, sans que nous puissions deviner ses soucis ni l'aider,...".5 D'autre part, au moment où les deux frères sont entièrement séparés, Louis et Alexis ont une relation un peu plus forte : "Autrefois Louis Tutoyait Alexis, comme cela se pratique entre cousins germains, mais depuis qu'ils étaient dans le même lycée, lui étudiant, l'autre aumônier, il s'efforçait d'éviter la deuxième personne. L'intimité dans laquelle ils avaient grandis, et qu'il aurait désiré voir se poursuivre et s'approfondir, c'était trouvée soudain brisée par cette relation nouvelle. Quant à Jean, il avait toujours été si lointain,... ". 6 Cet enfermement et cet isolement pousse Alexis à avouer à Louis qu'il ne connaît pas si son frère Jean aime la musique ou non: "A vrai dire je ne sais pas trop ; je n'ai jamais eu l'occasion de lui poser la question ; pourquoi ?"7

Il résulte de tout ceci qu'ils sont tous les quatre comme des étrangers qui vivent ensemble, dans des cases fermées : "Ils sont quatre solitaires qui se rendent parfois visite, et se retrouvent aux repas ". ${ }^{8} \mathrm{Au}$ deuxième étage, la famille Mogne vit en vase $\operatorname{clos}^{9}$, les relations entre les membres de cette grande famille sont presque les mêmes relations dans les autres étages, des isolements, des enfermements et des solitudes malgré que cette famille se compose d'onze personnes. Ces personnages ne se rencontrent que lors de leur diner rituel et routinier chaque soir. Dans cet extrait le père Frédéric Mogne résume les rapports entre les membres de cette famille : " Encore un peu de jour amer et violet. Etroit, restreint, l'espace dans lequel il rentre --- tant de personnes s'y coudoient, --- sans nouveauté --- on n'a ni le temps ni la place de les regarder. On arrive, on se retrouve, on dine. On s'évite, on ne sait rien de dire, on s'endort, tandis que tout continue à s'agiter, et le lendemain il faut retourner à la banque où l'on dit de lui que c'est un homme de quarante ans qui en paraît soixante,..."10

Un autre passage met l'accent sur la souffrance, et la solitude des habitants dans cet immeuble, cette fois, il nous décrit l'isolement et l'enfermement d'Elisabeth Mercadier, qui habite au sixième étage avec les ouvriers et les étudiants. Cette femme souffre d'une séparation totale avec le réel, elle vit dans ses fantômes et dans ses rêves: "Au-dessus des de Vere : Elisabeth Mercadier, toute seul à l'étage des chambres, puisque l'employé du métro et la vendeuse sont partis faire une petite bringue au restaurant. Elle a mangé avec les fantômes de ses anciens maîtres, qu'elle identifie dans son souvenir avec Saint Joseph et la Sainte Vierge. Comme tous les soirs elle espérait qu'ils allaient lui dire: demain l'enfant Jésus viendra partager votre repas, mais non, c'est toujours le même air désolé : il aimerait venir bien sûr, mais il a tant de travail en ce moment. "11 


\section{L'espace et l'homme dans les romans de Michel Butor}

Les habitants de cette maison parisienne ne se connaissent presque pas. Dans cet extrait, Frédéric Mogne et sa femme Julie ne connaissent même pas le nom de la fille qui habite au quatrième étage. Cette ignorance reflète en fait, la déchirure et l'enfermement qui prédomine sur les rapports entre les habitants de cette maison, en outre, il représente les relations faibles et fragiles entre les gens dans la société française dans les années quarante : "Les gens du quatrième, Les Vertigues font un grand tralala en l'honneur des vingt ans de leur fille; comment s'appelle-t-elle déjà, Julie ? Je n'en sais rien, ma foi ; il faudrait demander aux enfants ". ${ }^{12}$ Un autre exemple mérite d'être signalé à cet égard, Mme Lydie la mère d'Angèle Vertigues ne connaît pas son voisin Samuel Léonard qui habite au troisième étage : "Vous connaissez Samuel Léonard? Faisant effort pour fouiller dans sa mémoire : Samuel Léonard? Enfin, chère Lydie, il habite au-dessous de chez vous. Ah, je n'y étais pas du tout. Je l'ai rencontré deux ou trois fois dans l'escalier. "13

En outre, Michel Butor met l'accent aussi sur les fausses relations et l'émulation entre les frères. A titre d'exemple, les deux frères Mogne, qui habitent ensemble dans le sixième étage. Gérard Mogne refuse de prêter son frère Vincent une lame de rasoir : "Oui....Qu'est-ce que tu veux encore? Ouverture violente, croisement de raies claires sur le sol du couloir. Tu as une lame de rasoir? Écoute, mon vieux, tu es assommant; tu ne peux jamais avoir tes affaires. Demande à ta mère de t'en acheter; j'en ai plein le dos de te servir d'intendant." 14

Également, Butor représente la corruption morale entre les habitants de cet immeuble. Cette corruption est représentée dans la relation homosexuelle entre Samuel Léonard et son boy égyptien Ahmed, et Vincent Mogne et Ahmed : Croyez bien qu'il ne lui fallut pas longtemps pour se faire une idée fort précise des rapports qu'entretiennent son patron et son boy, car s'il a tout fait pour les dissimuler, particulièrement de sa nièce,... "15, "Le scandale et l'effroi sur ce visage qui cherche à se composer; lui aussi comprenait. Gérard murmura d'une voix presque naturelle : vous cherchiez quelqu'un? - Non, non, je vous remercie, c'est bien, c'est très bien. Et il répétait en s'en allant, comme une machine : C'est bien. "16

Dans un exemple marquant, Michel Butor, nous décrit la fausse relation entre le riche juif Samuel Léonard, et sa fausse nièce Henriette. Il entre dans la chambre d'Henriette dans le but de savoir ses nouvelles, les deux personnes ne se regardent pas face à face, mais d'une manière bizarre qui indique l'ambiance générale de la nature des relations familiales et sociales dominantes dans cette maison: "Il s'approche pour la caresser, sans qu'elle y prête attention. (...). Les deux têtes l'une contre l'autre se regardent dans leurs images. "17

Il est à noter également que l'enfermement des personnages dans cet immeuble n'est pas seulement un enfermement physique mais il est symbolique, puisqu'ils ont des consciences bornées et aliénées. ${ }^{18}$ "Ces personnages soumis à une convention qu'ils n'ont pas le courage de violer, se condamnant ainsi eux-mêmes à une infinie répétition dans l'orbe $d u$ mensonge". ${ }^{19}$ Pour conclure, et dans une expression évocatrice, Michel Butor résume fort bien que, les habitants de cet immeuble parisien, sous le titre : "D'animal fabuleux. " 20

En ce qui concerne L'Emploi du Temps le thème de l'enfermement est fortement évoqué également par Michel Butor, puisqu'il réussit à implanter son personnage- 
narrateur dans un labyrinthe spatial interminable, dans un exil atroce qui s'appelle Bleston. Dans ce passage Michel Butor nous explique fort bien la structure spatiale, labyrinthique de L'Emploi du Temps : "(...) nous avons la ville qui est considérée comme un lieu clos, mais ce lieu clos ouvre par des fenêtres intérieures sur un autre lieu clos qui est la terre, il y a un autre lieu clos très important et qui est la chambre dans laquelle se fait toute l'écriture, et à l'intérieur de la chambre il y a encore un autre lieu clos qui est la page et l'ensemble des pages qui formeront finalement le livre. " 21

$\mathrm{Vu}$ que dès les premiers instants de son arrivée à Bleston, Revel remarque que: "Les lueurs se sont multipliées". ${ }^{22}$ Cette multiplicité des lueurs nous parait comme un essor de ce labyrinthe spatial dès la première page de ce roman. Dans cette perspective, L'Emploi du Temps nous renvoie au roman de Robbe-Grillet, DANS LE LABYRINTHE, où il nous relate dès l'incipit du roman les chroniques de l'errance d'un soldat: "Je suis seul ici, maintenant, bien à l'abri. Dehors il pleut, dehors on marche sous la pluie en courbant la tête. "23, ce soldat: "Ne sait pas luimême où il va?"24

Dès son arrivée, Revel souffre à cause de la puissance : "Surtout maléfique ". ${ }^{25} \mathrm{de}$ Bleston. Ses malheurs commencent très tôt, puisqu'il ne prend pas : "Le grand train direct, celui que j'aurais dî prendre, (...), si j'avais su à quel point son heure d'arrivée était incongrue dans la vie d'ici,..." ${ }^{26}$, par conséquent, il est seul à la gare de Bleston, c'est le premier indice de sa solitude et de son enfermement: "Le chauffeur de taxi, dont j'étais le dernier espoir pour la nuit, m'a demandé où je voulais être mené (ses paroles ne pouvaient avoir d'autres sens), mais les mots qu'il employait, je ne les reconnaissais pas, et ceux par lesquels j'aurais voulu le remercier, je ne parvenais pas à les fermer dans ma bouche,...

Il trouve une grande difficulté à communiquer avec les gens à Bleston, c'est pourquoi, il évite toutes sortes de discussions par cette langue ce qui approfondit sa sensation de solitude: "Alors j'ai jeté un coup d'œil sur le compteur, et je l'ai payé largement afin d'éviter toute discussion,..."28, ou lorsqu'il veut manger, il trouve le même problème à capter les termes nécessaires: "J'aurais voulu dire: "je vous prie", mettre de l'amabilité dans ma demande, mais j'avais déjà le plus grand mal à retrouver les quelques substantifs indispensables"29, de même, quand il tend à demander l'adresse de son travail chez Matthews and Son: "J'ai préparé soigneusement mes mots pour interroger la demoiselle en bas. "30

En outre, malgré son inquiétude de suivre cet homme noir jusqu'à sa maison. Il accepte son invitation de prendre le thé chez lui sous prétexte qu'il: "Parlait si lentement que je le comprenais, et en même temps si mal qu'en adressant à lui, je n'avais plus aucune honte de prononciation détestable". ${ }^{31}$ Il est à noter que, le premier homme, qu'il rencontre à Bleston, c'est Horace Buck : "(...), son visage de même noir que l'eau." "Avec lequel j'ai eu une conversation personnelle dans cette ville,... ${ }^{» 32}$

Horace Buck est un étranger, exilé et nègre qui souffre de toutes sortes de racisme et de négligence dans cette ville. Cette rencontre met l'accent sur le problème de l'identité, et sur la souffrance des étrangers à Bleston. Cet étranger travaille à Bleston depuis dix ans, dans une usine de coton. Il soutient Revel lors de 
son séjour à Bleston. Revel écrit le mercredi 18 juillet qu'Horace est : "Mon sauveur" ${ }^{33}$ Horace Buck évoque en fait des problèmes très importants. Il souffre comme tous les africains d'un racisme éclatant, à cause de sa couleur: "Horace Buck est le type même de l'exclu. Comme noir il est ignoré des blancs et l'esprit provincial des noirs exilés le fait rejeter de leur communauté,... ". ${ }^{34}$ Cet extrait entre Horace et Revel met l'accent sur ce point: "Pourquoi ne voulez-vous pas accepter ce que je vous ai donné? Cela vous fait honte, qu'un homme de couleur vous ait payé à déjeuner?"35

Un exemple éclatant du racisme contre les étrangers et notamment les noirs à Bleston, lorsque Horace Buck a trouvé la nouvelle chambre pour Revel chez Mme Grosvenor. Cette dame n'aime pas les étrangers et précisément les noirs : "Elle voue tous ces black devils, (...), elle avoue tous ces démons noirs au même enfer, à la même potence, ne parvenant pas à comprendre pourquoi le gouvernement de Sa Majesté laisse les ports de l'ile ouverts à ces faveurs à figure à peine humaine,... ". 36 Cette xénophobie pousse le noir Horace Buck à se borne à jouer le rôle d'intermédiaire entre son ami Revel et Mme Grosvenor pour signer le contrat de la location de cette chambre. Plus tard, et à cause de cela, Horace Buck a brulé la boutique «Amusements » (pp.282-283), le dimanche 8 juin comme une réaction contre l'hostilité de la ville et de ses habitants.

Rappelons aussi que Michel Butor a évoqué le problème de l'exil et de l'esclavage tout au long de son premier roman Passage de Milan. Dans le premier étage, Charlotte Tenant dit: "Qu'elle était venue toute enfant"37 de 1'Allemagne. Dans le centre de l'immeuble, chez Samuel Léonard, le boy égyptien Ahmed est très mal traité, puisque, quand Henriette la fausse nièce de ce riche juif : "Le prend à la fois comme un domestique et comme un frère. L'oncle aimerait plutôt qu'elle soit dure; s'il le blâme sur la maison, c'est souvent quand ils sont ensemble; elle en a pitié, elle en est confuse, comme une enfant trouvée adoptée par un prince, qui continuerait à se juger indigne de si beaux valets. "38

Revenons de nouveau à L'Emploi du Temps, où L'Ecrou un des indices de la clôture et de l'enfermement dans le roman. L'Ecrou est l'hôtel consacré au séjour de Revel lors de son exil à Bleston. Cette chambre qu'il: "S'efforçait de ne pas regarder, une fois qu'il y était entré". ${ }^{39} \mathrm{Ce}$ nom est si : "Evocateur" 40 , de ses malheurs et de ses chagrins: "Vous allez à L'Ecrou, évidemment; le vieux Matthews y expédie toujours les nouveaux arrivants; c'est devenu presque un proverbe parmi nous. ". ${ }^{41}$ Cette chambre sordide nous renvoie bien évidemment à la chambre de son séjour à Minieh en Égypte, dans la mesure que, les deux chambres sont presque vides, elles donnent l'impression de la prison. Dans la chambre-prison de Bleston: "Il n'y avait pas de table; la fenêtre donnait sur un mur de briques au fond d'une cour". ${ }^{42}$ La chambre de Minieh est beaucoup plus sombre et plus modeste: "C'est ainsi que j'ai fait la connaissance de Hassan qui m'a servi en quelque sorte d'intendant pendant tout mon séjour et qui m'a fait enterrer dans une chambre vide, carrelée, haute de plafond, aux murs de ciment peints en vert tendre, qui, jusqu'à un certain niveau, se sont rapidement ponctués de petites taches de sang, parce que nous étions en automne, que le Nil commençait seulement sa décrue, et qu'il y avait par conséquent de nombreux moustiques très agaçants que j'écrasais avant de m'endormir"43" (...), je n'ai pas eu non plus de difficulté pour trouver des 
chaises, (...), je considérais qu'il m'était impossible de vivre sans table, en ayant besoin pour lire et écrire mon métier de professeur élémentaire qu'il fut, en ayant besoin même pour manger à mon aise." ${ }^{\prime 4}$

Lors de son entrée dans cette chambre néfaste il prend la décision de laisser cet exil : "Je ne puis pas rester ici, je ne dois pas rester ici, je suis perdu si je reste ici, dès demain je vais me mettre en quête d'un logement meilleur "45, "Dans ce recoin dont je désirais tant partir, dans cette chapelle de mesquinerie,... ". ${ }^{46}$ Ainsi, il réalise tôt que: "Dès que j'aurais quitté L'Ecrou, dès que je serais installé... ". ${ }^{7}$ Il s'efforce de franchir cet écueil. Il se met à chercher à une autre chambre plus confortable, donc il achète le journal l'Evening News : "C'est dans le but d'éplucher les, (ses), annonces ${ }^{» 48}$, mais ces premières tentatives échouent.

Finalement, grâce à son ami Horace Buck il trouve une autre chambre plus commode, le 37 Copper Street, dans un quartier central pas loin de son travail. Cette nouvelle chambre: "Est positionnée par l'expression affective" ma maison", signe d'une intimité et d'un vrai chez-soi retrouvés; elle est en outre déterminée grammaticalement par le possessif ce qui contraste vivement avec le sommaire plan de la ville, forcement abstrait et démarqué des plans imprimés. "49

Dans ce contexte, Henri Mitterrand remarque fort bien que, L'Ecrou, l'ancien hôtel de Revel est déterminé grammaticalement par l'article défini ce qui convient certainement à son attitude de Bleston:" (...),Madame Grosvenor m'a fait entrer pour la première fois dans cette chambre, que j'ai compris que j'avais enfin trouvé, que j'allais m'installer, prendre assise et appui, que c'en était fini avec ces randonnées harassantes et désespérantes d'un bout à l'autre de la ville à la recherche d'une case, (...) "50, "Ce n'est que peu à peu, lentement, m'apercevant de la clarté de cette chambre, de sa propreté, de la blancheur de son plafond, de la fraicheur de ses tentures, (elle venait d'être retapissée), de l'égarement d'avoir Dew Street en face de sa fenêtre, et non la façade sordide de quelque autre maison de Copper Street, ... " 51

La difficulté du déchiffrement de la ville de Bleston est l'un des motifs principaux de l'égarement et de l'exil de Revel à Bleston. Cette ville a une : "Structure indéterminée". 52 Cette structure imprécise mène à l'égarement et à l'errance de Revel au dédale de cette ville maudite : "J'avais compris que Bleston, ce n'est pas une cité bien limitée par une ceinture de fortifications ou d'avenues, se détachant ferme sur le fond des champs, mais que, telle une lampe dans la brume, c'est le centre d'un halo dont les franges diffuses se marient à celle d'autres villes. "53

Dans cette perspective, et à la fin de son séjour, Revel avoue qu'il est dans une grande geôle appelée Bleston : "(...), Bleston, dans, notre année, parce qu'il ne me reste plus que neuf jours avant de quitte tes murs de fonte, tes murs de pluie, tes murs de braise étouffée, ..." "54

Cette structure floue de Bleston est représentée aussi par l'image de l'œuf. Cette composition particulière de la ville en forme d'œuf où il n'y a pas de limites claires entre les endroits et entre les quartiers: "J'étais renseigné sur la structure de Bleston que n'aurait pu l'être un aviateur la survolant, ne serait-ce que par cette ligne pointillée marquant les limites de son territoires administratif en dehors 


\section{L'espace et l'homme dans les romans de Michel Butor}

duquel les maisons se groupent sous d'autres noms, la dessinant en forme d'œuf, la pointe au nord". ${ }^{55}$ Ce thème de l'œuf sert également comme un : "Symbole à la fois de la clôture. ${ }^{* 56}$

Un autre indice très remarquable dans cette optique, le thème de la foire mobile dans cette : "Ville mobile "57" Qui fait le tour de ton noyau". ${ }^{58}$ Ce thème confirme le concept de la structure nébuleuse de Bleston. Il est à noter au premier abord que, la ville de Bleston se compose de douze arrondissements, ainsi cette foire occupe chaque mois un quartier différent dans la ville. Ce thème de la foire est répété plus de quarante-cinq fois tout au long du roman. Ce grand nombre de répétitions reflète l'intérêt accordé par l'auteur à préciser la mobilité de cette ville et son impact sur le personnage-narrateur dans le roman.

Cette foire prend en fait, un itinéraire particulier selon un schéma singulier correspondant au style labyrinthique de l'auteur : " (...), Le terrain vague sur lequel était la foire au mois de janvier, sur lequel elle va se réinstaller dans quelques jours pour y demeurer tout au long de septembre,... "59 "(...), cette petite ville mobile un peu moins morose qui fait le tour de la grande en huit mois, s'arrêtant quatre semaines dans chacun des arrondissements extérieurs à l'exception du douzième où elle serait trop proche de Plaisance Gardens, cette grande foire immobile, $s$ 'arrêtant toujours dans les mêmes terrains vagues,... "60

Ainsi, cette foire ambulante dans la ville de Bleston selon le schéma butorien : "Est absente de quatre arrondissements sur douze : le IV", le quartier des gares, (...), le VII , où se trouve la Boutique des Amusements, (...), le VIII le quartier de l'Ancienne Cathédrale,(...), le XII où se tient la foire immobile, de celle de Plaisance Gardens". ${ }^{61}$ Pour conclure, cette structure imprécise et monotone de l'espace butorien implique: "Un temps indiffèrent, vide, mort, un temps qui n'avance pas, qui n'a pas d'avenir. " 62

Dans La Modification l'espace de même que le temps joue un rôle capital à la transmutation ultime de la décision de Delmont puisque selon Butor : "Les lieux ayant toujours une historicité, soit par rapport à l'histoire universelle, soit par rapport à la biographie de l'individu, tout déplacement dans l'espace impliquera une réorganisation de la structure temporelle, changement dans les souvenirs ou dans les projets, ... ${ }^{\star 63}$

Revenons de nouveau au thème de la clôture. Dans La Modification, Delmont subit également l'exil et l'enfermement comme Revel dans le roman précédent. Si Revel est exilé à l'étranger pendant un an. Delmont subit aussi l'enlisement et la solitude, mais cette fois dans sa maison et entre ses enfants. Il n'est pas un captif d'un exil spatial seulement mais il est aussi un enfermé dans ses pensées et ses préoccupations interminables lors de son trajet Paris-Rome. Sa maison est dominée par les fausses relations, puisqu'il est très loin de ses enfants et de sa femme. Sa relation avec ses enfants est très fade et insipide, il sent continûment qu'il est étranger même dans sa maison et entre ses quatre enfants : "Vous ne comprenez pas ce qu'ils pensent, vous ne comprenez pas ce qu'ils aiment, ils forment une espèce de ligne contre vous tous les trois,... "64 "(...) vous subissez leur chahut, ce qui vous agace contre eux, ce qui les empêche d'avoir confiance en vous, de telle sorte qu'ils sont devenus pour vous de petits étrangers sauvages, audacieux et complices, qui se doutent fort bien que quelque chose ne va pas entre leur mère et vous ". ${ }^{65} \mathrm{Il}$ souffre 
aussi, avec sa femme qu'il n'aime pas, alors il décide finalement de mettre fin à cette relation douloureuse et pénible : Elle attendait que vous l'embrassiez comme toutes les autres fois, mais vous vous refusiez à prolonger plus longtemps cette comédie, ... ${ }^{" 66}$

Lors de son séjour à Paris il est dans une grande prison, sa femme, son travail et ses ennuis poussent ce misérable à vivre comme un touriste chez lui. Lorsqu'il décide de délaisser cette vie auprès de sa femme. Il entre dans un autre enfermement spatial très étroit. Le compartiment du train ou bien dans: "Cette case, votre domicile dans l'espace de ce train qui vous emporte, marquée par cet objet vous appartement, votre valise gauche sur le filet,... ". ${ }^{67}$ Pendant son escapade à Rome, Delmont craint d'entrer dans son compartiment afin d'éviter ses agaces et ses ennuis: " (...), Hésitez-vous à entrer dans ce compartiment comme si toutes ces pensées de tout à l'heure allaient se ressaisir de vous dès le moment où vous serez assis de nouveau à cette place que vous avez choisie au départ comme celle qui vous revient ?"68 Il reste dès la première page de ce roman enfermé comme un prisonnier ou comme un captif de cette cage mouvante, de ses pensées et de ses préoccupations. Il vit avec ses anciens cauchemars avec Henriette et ses prochains progrès avec Cécile: "(...) , si vous n'aviez pas ce salut, Cécile, si vous n'aviez pas cette gorgée d'air, ce surcroît de forces, cette main secourable qui se tend vers vous messagère des régions heureuses et claires, depuis cette lourde ombre tracassière dont vous allez pouvoir enfin vous séparer de fait, jusqu'à cette magicienne qui par la grâce d'un seul de ses regards vous délivre de toute cette horrible caricature d'existence, vous rend à vous-même dans un bienfaisant oubli de ces meubles, ... "69

Cette solitude de Revel et de Delmont nous renvoie bien évidemment à celle de Pierre Vernier dans Degrés: "(...), tu t'es mis à penser à cette année qui allait recommencer, à cette vie de solitude et de stérilité qui t'attendait. Il y a avait pour toi deux moyens d'en sortir : la littérature ou le mariage ". ${ }^{70}$

En fait, il excelle à présenter cet enfermement et cet emprisonnement dans tous ses romans, par certains thèmes dominants, à titre d'exemple, l'emploi systématique des fenêtres et la technique du : "Clair-obscur ". ${ }^{71}$ Commençons à première vue, par le thème de la fenêtre qui est considéré : "Le premier indice de la clôture ", ${ }^{72}$ dans les romans de Butor, puisque la fenêtre est le moyen unique entre les personnages butoriens et l'extérieur. Ainsi: "Cette fermeture de l'espace mène toute la narration" $^{\prime 73}$, dans Passage de Milan et dans les autres romans de Butor. Dès la phrase-incipit de Passage de Milan, qui commence par l'abbé Jean Ralon penche à sa fenêtre contemplant la ville de Paris: "L'abbé Ralon se pencha à la fenêtre. Il y avait Paris tout autour, séparé par une fausse muraille de brumes et de fumées couleur de teinture d'iode....". ${ }^{74}$ Ce thème est répété tout au long du roman comme un indice révélateur de l'enfermement intact des individus butoriens. Dans cet exemple le père Paul Mogne raconte à son frère Fréderic Mogne qu'il ne sort pas à cause de son âge et de sa faiblesse. Il considère sa fenêtre comme un sauveur de cet enfermement mortel ou bien pour respirer l'air pur: "Je sortirai de moins en moins, Fréderic, je ne sortirai presque plus. Mais quelle idée ? L'été va venir, tu te sentiras beaucoup mieux (...), Il n'insiste pas. Il ouvre la fenêtre aspire l'air du dehors, tire les volets, ferme doucement, sort discrètement, promené son désœuvrement. "75 


\section{L'espace et l'homme dans les romans de Michel Butor}

Les fenêtres restent fermées tout au long du roman. Puisqu'il semble que les habitants de cet immeuble se bornent à utiliser ses fenêtres comme des miroirs : "Mettez-vous à la porte, vous aurez la fenêtre fermée devant vous, avec ses rideaux qui l'encadrent, et tranchant sur les filets des vitres, les cheveux noirs de Félix qui ne sait quelle sœur regarder ". ${ }^{76}$ Dans le premier étage où l'isolement est complet entre les personnages. L'abbé Alexis vit dans une solitude quasi intacte : "La flamme s'est reflétée dans la fenêtre, et dans la glace qui lui sert à se raser,... ". ${ }^{77} \mathrm{C}$ 'est pourquoi, lorsque Mme Tenant laisse sa fenêtre ouverte, elle considère cela comme un mauvais présage : "La fenêtre était restée ouverte, et dans sa préoccupation elle ne s'en était pas aperçue; presque un mauvais présage, n'estce pas? Elle s'était levée toute émue pour la fermer. "78

Dans L'Emploi du Temps, le thème de la fenêtre prédomine tout le roman aussi, comme un emblème de l'enfermement et de la solitude de Revel à Bleston. Avant de son arrivée à Bleston Revel regarde à travers la vitre du train : Dans ce coin de compartiment où j'étais seul, face à la marche, près de la vitre noire couverte à l'extérieur de gouttes de pluie, myriade de petits miroirs,... ". ${ }^{79}$ Lorsqu'il entre chez Matthews and Son pour la première fois il fait attention que la seule place vide est : "La deuxième table, près de la fenêtre,... ". ${ }^{80}$ Butor insiste pour la deuxième fois à préciser la place de Revel, chez Matthews and Son : "Au coin, près de la fenêtre ". ${ }^{81} \mathrm{Au}$ restaurant avec son ami de travail Lucie, ils occupent la place près de la fenêtre: "James et moi, après ce court spectacle, près d'une fenêtre au premier étage de l'Oriental Rose,....". ${ }^{82}$ Cependant, nous réalisons la valeur de la fenêtre pour Revel quand il arrive pour la première fois à L'Ecrou avec James Jenkins, à ce moment Revel prend la décision de modifier cette chambre sordide : "(...), La fenêtre donnait sur un mur de briques au fond d'une cour". ${ }^{83}$ Tandis que sa nouvelle chambre a une fenêtre qui donne à Dew Street: "(...), à travers de la fenêtre, peu à peu, paraissait Dew Street.

D'après ce qui précède, il nous semble que, Revel reste lors de son séjour à Bleston, dans une recherche perpétuelle d'être près de la fenêtre, dans son travail, son domicile, ses repas dans les restaurants et dans les bus. Puisqu'elle est le seul moyen pour cet étranger de contempler cet univers extérieur.

Dans La Modification, ce thème reste dominant également, parce que le personnage-narrateur nous raconte tout au long du roman ses préoccupations dans sa prison mouvante ; le compartiment du train. Ce coin dans la case du train est le seul lien entre ce prisonnier et l'extérieur. Il reste pendant presque vingt-deux heures, en répétant ce phrase-thème, "de l'autre côté de la fenêtre, ou au-delà de la fenêtre ", cette phrase qui prend le lecteur du roman loin de la case du train, et loin de cet homme marri et de ses angoisses effrayantes. Ce thème sert également à décrire l'espace extérieur dans le roman. A titre d'exemple : "Au-delà de la fenêtre toujours aussi brouillée de pluie, se superposant à la série de pylônes réguliers comme un coup inattendu légèrement plus fort, ... "85

Dans le but d'éviter la répétition de la même phrase ou bien du même terme. Butor recourt aux termes analogues comme; «à travers de la vitre », pour transfigurer la direction de la description, ou pour orienter le lecteur à un espace plus vaste et plus différent : "Puis quelqu'un demandera d'éteindre la lampe. A travers la 
vitre un peu moins brouillée par les gouttes de la pluie qui s'atténué, vous apercevez une voiture semblable à la vôtre, une quinze chevaux noire toute maculée de boue, ... ". ${ }^{86}$ En outre, il utilise la vitre du train comme: "Un écran transparent, elle permet de dévoiler une réalité dont on sera séparé ; les éléments qui vont apparaître alors dans cet écran acquièrent un statu à la fois objectif et poétique car ils vont former une sorte de tableau que la vitesse du train anime "87, comme dans ce passage :"(...), dans la fenêtre en face de vous votre image qui se balance comme celle d'un homme ivre prêt à tomber, jusqu'au moment où, à travers les nuages qui se séparent, la lune apparaît et vous efface ". ${ }^{88}$ De même, il utilise le thème de la fenêtre comme un reflet de l'état de ses personnages.

A cet égard nous estimons indispensable de comparer ces deux extraits. Le premier lorsque Delmont souvient les derniers moments avec sa femme Henriette avant son départ. Il était marri et mécontent de sa vie avec sa femme, dont la fenêtre est fermée: “(...), Les draps qui émergeaient de l'obscurité semblable à des fantômes vaincus, écrasés au ras du sol mou et chaud dont vous à vous arracher. Tournant vos yeux vers la fenêtre, vous avez vu les cheveux autrefois noirs d'Henriette, et de son dos se détachant devant la première lumière terne et décourageante, doucement, brusquement, au travers de sa chemise de nuit blanche un peu transparente, ... $" 89$

L'autre extrait est lorsqu'il vit dans la nostalgie de Rome et de l'amour de sa maîtresse, dans ce cas-là la fenêtre est ouverte: "Vous vous êtes installé dans le fauteuil du salon, près de la fenêtre où apparaît si admirablement la frise illuminée du Panthéon, à écouter à la radio quelques extraits de l'Orfeo de Monteverdi ;... ". 90 Un autre exemple dans ce propos mérite d'être signalé lorsqu'il décrit l'espace extérieur de son compartiment. Cette description reflète l'état confus et affligé de Delmont: "Devant la fenêtre qui donne sur les champs rapides et le brumeux horizon lent,... "91

Il résulte de tout ceci que, la position de la fenêtre correspond d'une façon ou d'une autre à l'état psychologique de Delmont dans le roman. La fenêtre dans toutes les œuvres butoriennes est un symbole de la clôture et de l'enfermement. Elle est considérée comme la seule voie entre les individus et le monde extérieur. Elle tient aussi une dimension mythique, comme dans Passage de Milan, Butor dit que : La vitre est en effet une image qui m'est chère. Elle peut ou non être transparente : elle peut ou non me faire voir au-delà d'elle. Vitre ou miroir, c'est le symbole du roman lui-même; il a sa propre dureté et à travers lui, je plonge, je découvre autre chose. ${ }^{" 92}$

Au même titre, Michel Butor utilise méthodiquement la technique de l'éclairage afin de mettre l'accent sur l'isolement, la mélancolie et l'enfermement des individus dans ses romans. Prenons comme point de départ Passage de Milan, où l'obscurité est le thème dominant sur tous les appartements, sur tous les coins même sur l'escalier de cette maison. Butor insiste au début de son roman à orienter son lecteur dans un édifice mal éclairé, c'est pourquoi, il commence par l'escalier : "La verrière qui servait de plafond à la cage de l'escalier n'éclairait pour ainsi dire plus ". ${ }^{93} \mathrm{Au}$ premier étage Alexis vit dans une chambre : "Obscure ". ${ }^{94}$ Restons au premier étage, dans cet extrait, Charlotte Tenant, la servante allemande qui habite chez les Ralon 


\section{L'espace et l'homme dans les romans de Michel Butor}

connaît la place par cœur, puisque : "Dans l'obscurité même elle aurait su trouver le piton de la râpe,..." ". ${ }^{95}$ Les habitants du sixième étage sont dans une obscurité : "Quasi-totale, même à midi, et, de nuit, par trois faibles lampes,... ".96 L'un des exemples les plus frappants se trouve à la page (p.48), lorsque Butor nous décrit cette obscurité presque totale dans le deuxième étage. Cette obscurité permet de voir à peine les visages de la famille Mogne lors de leur diner rituel. Il nous paraît que cet exemple reflète parfaitement la réalité de la société française de cette époque, où les individus sont serrés, mais ils ne se regardent pas : "On est serré. Les angles de la table envahissent l'espace à tel point qu'on a presque toujours peine à voir le visage entier d'un de ses interlocuteurs." ${ }^{97}$ Ainsi chez les Mogne : "La salle est pour l'instant la seule partie de l'étage qui soit vivante. Toutes les autres pièces sont obscurées,... ".$^{98}$ Même au cinquième étage, la lumière est très faible, pour contempler le tableau de Martin de Vere : "Maurice Gérard contemple le tableau qui lui fait face, mal éclairé par la lampe,... "99

En définitive, il nous paraît que : "Grâce à ce système d'éclairage, il y a souvent cette idée de l'impossibilité de voir la réalité complète de quoi que ce soit". ${ }^{100}$ En outre, cet espace sombre: "Associé à la clôture, suggère la claustrophobie. La vie dans cet espace offre l'image des rapports croisés, remplis de haine, de jalousie et de dénigrement mutuel. Les portes et les fenêtres closes, les pièces obscures et sans air créent cette impression de frustration, d'étouffement et de malaise. " 101

Cette obscurité prédomine aussi L'Emploi du Temps. Elle empêche cet étranger de voir ce qui se passe à l'extérieur de son compartiment: "Près de la vitre noir couvert à l'extérieur de gouttes de pluie, myriade de petits miroirs, chacun réfléchissant un grain tremblant de la lumière insuffisante qui bruinait du plafonnier Sali,... ". ${ }^{102}$ Cet extrait nous renvoie à l'abbé Jean Ralon dans la première page de Passage de Milan en contemplant Paris de la fenêtre de sa chambre au premier étage : "Il y avait Paris tout autour, séparé par une fausse muraille de brumes et de fumées couleur de teinture d'iode,... "103

En fait, Butor insiste dans ces deux exemples sur le rôle symbolique de l'éclairage à diffuser et à refléter la réalité de cette époque brumeuse pendant et après la seconde guerre mondiale. Ce thème de l'obscurité est lié tout au long du roman à l'exil et à la pauvreté des individus de Bleston. Signalons que lors de sa première visite pour la chambre d'Horace Buck, il entre méfiant : "Dans la chambre obscure où je n'ai distingué d'abord que le grand lit défait,... ". ${ }^{104}$ Dans L'Ecrou son logement misérable à Bleston, l'un des désavantages de cette chambre triste selon Revel est la faiblesse de son éclairage. Il remarque que cette chambre est: "Mal éclairée"105, "Le dimanche à L'Ecrou, de ces horribles retours, de ces horribles soirées mal éclairées, qu'enfin, après être resté plus d'un mois dans cette antichambre,... "106

Cependant, sa nouvelle chambre autrement dit "sa nouvelle maison ", au sudouest de Bleston se caractérise en premier lieu, par sa clarté : "Ce n'est que peu à peu, lentement, m'apercevant de la clarté de cette chambre, de sa propreté, de la blancheur de son plafond, de la fraicheur de ses tentures,... ". 107 En outre, l'éclairage reflète aussi, l'état de la méfiance, et de la souffrance de ce prisonnier, lors de son séjour à Bleston: "Les jours n'auront déjà plus que cette pauvre clarté 
déchirante, cette clarté de larmes et de lamentation, qu'ils avaient au début d'octobre lors de mes premiers pas, de mes premières recherches,.. $\gg 108$

Dans La Modification, l'éclairage tient également un rôle essentiel à révéler l'état de Delmont, lors de son trajet dans le train entre Paris-Rome. Lors de ce trajet le compartiment du train est très mal éclairé : "(...), Dans ce compartiment vraisemblablement éclairé seulement par la veilleuse bleue par cette petite ampoule sphérique et sombre que vous apercevez à l'intérieur du lampadaire, nichée entre les deux autres transparentes à l'intérieur du lampadaire, nichée entre les deux autres transparentes piriformes?". ${ }^{109}$ L'image obscure prédomine la vie de Delmont, un exemple mérite d'être signalé à cet égard, lorsqu'il se réveille le jour de son départ, il voit que: "Les draps qui émergent de l'obscurité semblables à des fantômes vaincus, écrasés au ras du sol mou et chaud dont vous cherchiez à votre arracher. "110

Si l'obscurité est un sceau de la clôture et de la solitude dans les romans de Michel Butor, dans les œuvres de Balzac, l'obscurité est un indice de la cupidité des individus. Dans son roman, Eugénie Grandet, Balzac insiste tout au long du roman à représenter la radinerie de $\mathrm{M}$. Gandet. Balzac disait fort bien que : "Molière a fait l'avare, mais j'ai fait l'avarice" 111 , dans le même sens Petit de Julleville écrit que : "Grandet ne dit pas une seule parole, ne fait pas un seul geste qui ne manifeste son avarice". 112 


\section{L'espace et l'homme dans les romans de Michel Butor}

Bibliographie

1- Aziza Awad, L'immeuble parisien dans Pot-Bouille d'Emile Zola et dans Passage de Milan de Michel Butor : du chronotope au mythe, Paris, EDILIVRE APARIS, 2011, p.99.

2- Michel Butor, Improvisations sur Michel Butor, La Différence, 1963, p.71.

3- Georges Raillard, Butor, coll Union général d'Édition, 1974, p.9.

4- Michel Butor, Passage de Milan, Paris, Minuit, 1954, p.11.

5- Ibid.p.29.

6- Ibid.pp.41-42.

7- Ibid.p.45.

8- Ibid.p.44.

9- Skimao, Bernard Teulon-Nouailles, Michel Butor Qui êtes-vous ?, Lyon, La Manufacture, 1988, p.14.

10- Michel Butor, Passage de Milan, p.53.

11- Ibid.p.83.

12- Ibid.p.65.

13- Ibid.p.93.

14- Ibid.pp.30-31.

15- Ibid.p.58.

16- Ibid.p. 235 .

17- Ibid.p. 90 .

18- Skimao et Bernard Teulon- Nouailles, op.cit.p.53.

19- Georges Raillard, Michel Butor, Paris, Éditions Gallimard, 1968, p.52.

20- Michel Butor, Passage de Milan, p.88.

21- Bernard Valette, Entretien avec Michel Butor, in Analyses et réflexions sur Butor, L'Emploi du Temps, marketing, 1992, p.16.

22- Michel Butor, L'Emploi du Temps, Paris, Les Éditions de Minuit, 1956, p.9.

23- Alain Robbe-Grillet, Dans le Labyrinthe, les Éditions de Minuit, 1959, p.9.

24- Ibid.p.35.

25- Bernard Valette, Entretien avec Michel Butor, in Analyses et Réflexions sur Butor,

L'Emploi du Temps, op.cit.p.19.

26- Michel Butor, L'Emploi du Temps, pp.9-10.

27- Ibid.p.12.

28- Ibid.p.20.

29- Ibid.p.16.

30- Ibid.p.24.

31- Ibid.p.33.

32- Ibid.p.37.

33- Ibid.p.138.

34- Jean Roudaut, Michel Butor ou le livre futur, Paris, Éditions Gallimard, 1964, p.162.

35- Michel Butor, L'Emploi du Temps, p.33.

36- Ibid.p.139.

37- Michel Butor, Passage de Milan, p.19.

38- Ibid. pp, 136-137.

39- Michel Butor, L'Emploi du Temps, p.27.

40- Henri Mitterrand, L'Emploi du Temps, Paris, Nathan, 1995, p.28.

41- Michel Butor, L'Emploi du Temps, p.22.

42- Ibid.p.23.

43- Michel Butor, Le Génie du lieu, Paris, Grasset, 1958, p.136. 
44- Ibid.p.138.

45-Michel Butor, L'Emploi du Temps, p.23.

46- Ibid.p.72.

47- Ibid.p.51.

48- Henri Mitterrand, L'Emploi du Temps, op.cit.p.48.

49- Ibid.p.44.

50- Michel Butor, L'Emploi du Temps, p.140.

51- Ibid.p.141.

52- Henri Mitterrand, L'Emploi du Temps, op.cit.p.24.

53- Michel Butor, L'Emploi du Temps, p.44.

54- Ibid.p.383.

55- Ibid.p.54.

56- Henri Mitterrand, L'Emploi du Temps, op.cit.p.29.

57- Michel Butor, L'Emploi du Temps, p.135.

58- Ibid.p.374.

59- Ibid.p.309.

60- Ibid.pp.135-136.

61- Elise Jongeneel, Michel Butor, Le Pacte Romanesque, Librairie José Corti, 1988, p.136.

62 Viviane Rosé, La ville comme une figure de l'immonde, in Analyses et Réflexions sur Butor, L'Emploi du Temps, op.cit. p.41.

63- Georges Raillard, Michel Butor, op.cit.p.92.

64- Michel Butor, La Modification, Paris, Minuit, 1957 p.38.

65- Ibid.p.79.

66- Ibid.p.78.

67- Ibid.p.22.

68- Ibid.p.196.

69- Ibid.p.40.

70- Michel Butor, Degrés, Paris, Gallimard, 1978, p.162.

71- Aziza Awad, op.cit.p.96.

72- Ibid.p.102.

73- Ibid.p.101.

74- Michel Butor, Passage de Milan, p.7.

75- Ibid.p.9.

76- Ibid.p.53.

77- Ibid.p. 13 .

78- Ibid.p.20.

79- Michel Butor, L'Emploi du Temps, p.9.

80- Ibid.p.21.

81- Ibid.p.25.

82- Ibid.p.159.

83- Ibid.p.23.

84- Ibid.p.144.

85- Michel Butor, La Modification, p.99.

86- Ibid.p.102.

87- Henri Mitterrand, La Modification, Paris, Nathan, 1992, p.27.

88- Michel Butor, La Modification, p.99.

89- Ibid.p.16.

90- Ibid.p.82.

91- Ibid.p.25. 


\section{L'espace et l'homme dans les romans de Michel Butor}

92- Michel Butor, Entretiens, Quarante ans de vie littéraire, volume 1, Éditions, Joseph K, 1999, réunis, et présentés par Henri Desoubeaux, p.39.

93- Michel Butor, Passage de Milan, p.11.

94- Ibid.p.13.

95- Ibid.p.19.

96- Ibid.p.15.

97- Ibid.p.48.

98- Ibid.p.53.

99- Ibid.p.84.

100- Aziza Awad, op.cit.p.96.

101- Ibid.p.104.

102- Michel Butor, L'Emploi du Temps, p.9.

103- Michel Butor, Passage de Milan, p.7.

104- Michel Butor, L'Emploi du Temps, p.34.

105- Ibid.p.53.

106- Ibid.p.141.

107- Ibid.p.141.

108- Ibid.p.149.

109- Michel Butor, La Modification, p.32.

110- Ibid.p.16.

111- Honoré de Balzac, Eugénie Grandet II (extraits), préface de Félix Guirand, Paris, Librairie Larousse, 1934, p.107.

112- Ibid.p.106. 